\title{
Using Patient-Reported Measures to Improve Outcomes in Kidney Disease
}

\author{
Sofia Mclaren ${ }^{\mathrm{a}}$ Manisha Jhamb ${ }^{\mathrm{b}}$ Mark Unruh ${ }^{\mathrm{a}, \mathrm{c}}$ \\ aDivision of Nephrology, Department of Medicine, University of New Mexico, Albuquerque, NM, USA; ${ }^{\mathrm{b}}$ Renal- \\ Electrolyte Division, University of Pittsburgh Medical Center, Pittsburgh, PA, USA; ${ }^{C}$ Renal Section, New Mexico \\ Veteran's Hospital, Albuquerque, NM, USA
}

\section{Keywords}

Patient-reported outcomes - Health-related quality of life . Chronic kidney disease - Dialysis - Electronic health record

\begin{abstract}
Patients with CKD and ESRD are vulnerable to increased mortality rates and other poor outcomes. Among those with ESRD, their health-related quality of life (HRQOL) is shown little to no improvement as they undergo treatments such as dialysis and providers concurrently manage other health issues that complicate their already vulnerable state. This review synthesizes evidence demonstrating that a focus on measuring and monitoring patient-reported outcomes (PRO) such as pain and depression can improve HRQOL. Patient-centered care has the potential to create an efficient way for clinicians to address specific challenges facing patients. While there is an emerging literature assessing the use of PROs in kidney research, by examining relevant research in other disciplines it is possible to generate better ways to use PROs in this high-risk population. Electronic health records as well as various other electronic methods of communication between the clinician and patient may serve to accelerate the trajectory toward patient-centered care using PROs.

(c) 2021 S. Karger AG, Basel
\end{abstract}

\section{Introduction}

Health-related quality of life (HRQOL) is the patient's overall perception of the influence of a disease condition or its treatment on their life and well-being [1]. Patients with kidney disease have been observed to report severely impaired HRQOL throughout the course of the disease and value this as an important clinical and research outcome [1]. HRQOL is one of the many patient-centered outcomes that can be measured using patient-reported outcome (PRO) measures. PROs are self-reported data obtained directly from patients [2], focusing on outcomes such as well-being, overall health, symptoms, functionality, and life participation [2]. Patient-reported outcome measures (PROMs) are survey instruments that allow the patient to reliably report on a range of physical and psychosocial domains, without misinterpretation, and can be generic for different populations or can be diseasestate or symptom specific [3].

Incorporating PROs in clinical care is important for providing patient-centered care and potentially improving quality of life for patients. A challenge to this is how best to collect PROs in a practical and resource efficient manner that can be incorporated as part of routine clinical care. Electronic health records (EHRs) may provide a platform to incorporate PROs into clinical care in an efficient and secure way. EHRs can further provide oppor-

Correspondence to:

Mark Unruh, mlunruh@salud.unm.edu 
tunities for acting on the PROs to improve symptom management by providing relevant clinical information and decision support for providers, as well as self-management support and peer-community support for patients [4]. The goal of this review is to synthesize evidence demonstrating that a focus on measuring and monitoring PROs such as pain and depression can improve HRQOL of patients with ESRD.

\section{HRQOL in Patients with Kidney Disease}

HRQOL is impaired across the stages of advanced $\mathrm{CKD}$, and worsening decrements in HRQOL are associated with increasingly advanced disease [5]. Moreover, patients with CKD and other comorbid conditions such as diabetes have been shown to have HRQOL scores as low as patients with ESRD already undergoing dialysis [1]. Thus, the contribution of comorbidities to HRQOL is an important aspect to consider when using PROMs and designing interventions to improve HRQOL among the CKD population. Moreover, for ESRD patients on dialysis, treatment-related factors such as time spent on dialysis, frequency of dialysis, dialysis modality, and symptoms directly related to dialysis may be important contributors to their HRQOL.

\section{Measurement of PRO in Patients with Kidney Disease}

Patients with kidney disease have high symptom and comorbidity burden and increasing the use of PROs in nephrology clinical practice and research is important to improve patient-centered care and HRQOL. There are several PROs that have been used and validated among patients with kidney disease, although prior studies have largely focused on patients on dialysis. Some of these are generic, such as the Short Form-36 (SF-36), and some are more kidney and symptom specific such as measures of uremic pruritis. A systematic review found that the Kidney Disease Quality of Life-36 (KDQOL-36) had strong evidence for internal consistency and moderate evidence for hypothesis testing among patients with CKD. For dialysis patients, there was evidence to support the use of either the KDQOL-SF or the KDQOL-36 [6]. The KDQOL-36 has been recommended for clinical practice since it is slightly shorter than the KDQOL-SF, which has been largely used for clinical trials [7]. There is ongoing work to optimize the use of PROs in CKD to improve HRQOL by assessing the extent to which the KDQOL-36 reflects the contemporary patient experience and symptoms.

More recently, the National Institutes of Health-funded Patient-Reported Outcomes Measurement Information System (PROMIS) [8] provides a central repository with access to validated, precise PRO measures, focusing on physical, mental, and social well-being related to different chronic diseases [8]. These PROMIS questionnaires can be administered electronically using a computer-adaptive design to minimize participant burden and provide a way for cross comparison of PROs across multiple disease states. These are also part of the National Institutes of Health common data elements and are being increasingly incorporated into contemporary kidney clinical research studies.

There are not enough strategies to assist health-care providers in the use of PRO measures when evaluating kidney care. One approach aims to address PROs with the use of a realist synthesis approach, looking at a wide range of data, to create a kidney-specific program theory to optimize the use of PROs on individual and aggregate levels of patient-centered care [9]. There has also been a push toward collecting PROs electronically directly from the patients, and now, with a push toward telemedicine because of the COVID-19 pandemic, EHRs may become the best mode of communication between physician and patient. EHRs are efficient and secure, and the patient-facing portals of EHRs may provide an opportunity to collect PROs and offer better care for patients with chronic illnesses, including implementation of specific interventions that may improve patient HRQOL [4].

An important aspect of PRO evaluation is measurement of longitudinal changes in these outcomes, and EHRs can provide an efficient way to curate PROs at multiple time points. These data can provide trends for both patients and their providers, which can then be used to make clinical decisions for individualized patient-centered care. In addition, PROs collected through EHRs can also allow providers, practices, and health care systems to view and summarize data from multiple patients at once and allow for implementation of system-level practice changes that may improve management of PROs and HRQOL. Future research is required on the feasibility, usability, and effectiveness of EHR-based PROMs in renal/CKD and dialysis clinics and its impact on HRQOL of patients with kidney disease.

There are many benefits to measuring PROs, and it importantly provides the patient with a voice in their care and perhaps some peace of mind. For example, allowing patients to have a time of reflection and evaluation of 
their current state prior to an office visit could provide them a sense of support and reassurance that they are taking the right steps to improving their health. Although there may not be a direct or effective treatment for a reported symptom, the acknowledgment of the symptom by their physician can provide validation and make the patient feel heard. PROs can serve to foster communication and build a solid relationship between the provider and patient, which could lead to more effective treatment because the patient potentially feels more comfortable disclosing information about their health. PROs can also increase provider accountability and allow for comparisons of different providers' performances, which could lead to an improvement in quality of treatment by changing how it is organized and delivered [10]. This information could inform patients in selecting a provider and can be used to incentivize health system performance. Also, using PROs over time allows for the identification and monitoring of changes that occur over time in regard to the patients' health as well as attitude about their health. Finally, PROs are the first step to developing interventions because the voice of a patient who will be undergoing those interventions is the most important voice.

\section{Interventions to Improve HRQOL}

It is important for nephrologists to have the ability to treat patients holistically and not only target the biochemical markers of CKD. In addition to testing the impact of treating comorbid conditions among those with ESRD, there have been a range of trials demonstrating the effects of dialysis dose, membrane flux, dialysis timing, and frequency on PRO. One study tested the impact of an extended dialysis treatment on the primary outcome of HRQOL as assessed by the EuroQOL 5-Dimension (EQ5D) measure [11]. The trial observed that extending dialysis hours did not improve overall quality of life over a 12 -month period. The increased hours were shown to be potentially helpful with clinically small improvements in physical and mental component summary scores [11]. The findings of this study and others may suggest that treatments optimizing dialysis time may have modest impacts on PRO. Alternatively, treatment of comorbid conditions may present feasible and highly impactful approaches to improving PRO among those on dialysis. Depression is one of the most prevalent conditions in dialysis patients. Studies such as A Trial of Sertraline versus Cognitive Behavioral Therapy for End-stage Renal Disease Patients with Depression (ASCEND) are impor-

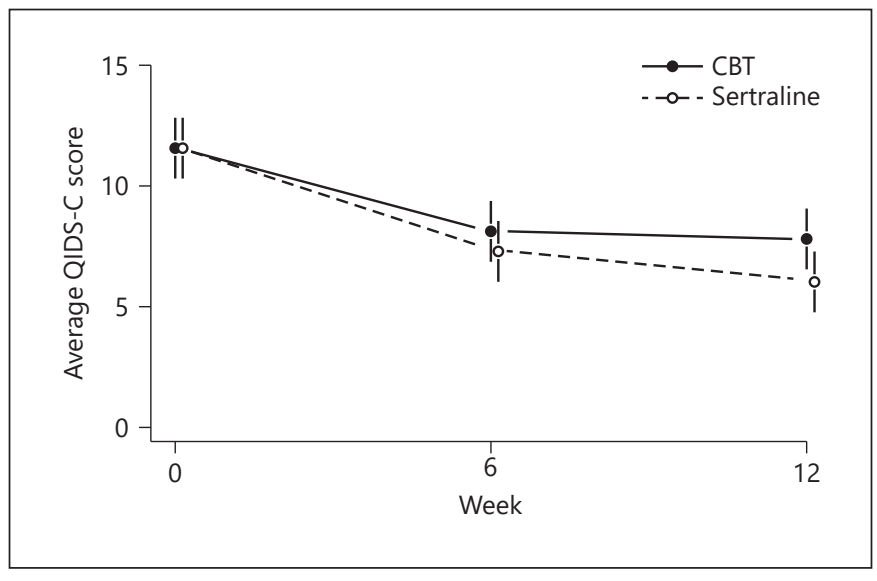

Fig. 1. Longitudinal data on the primary outcomes measure of QIDS-C scores among patients receiving hemodialysis with depression who were randomly assigned to $\mathrm{CBT}$ or sertraline treatment [11].

tant for the evaluation of management strategies for improving psychosocial health. This study used simple PROs to screen for depressive symptoms among patients undergoing thrice-weekly dialysis. Although the study found that sertraline was slightly more effective in improving depressive symptoms (shown in Fig. 1), sertraline led to the occurrence of more adverse events when compared to cognitive behavior therapy [12]. This study supports the position that PROs may be used effectively to measure and monitor symptoms and underscores need for symptom management strategies that can be offered and individualized for the patients [12] (shown in Fig. 1).

Several approaches used in oncology may also be considered for use among the ESRD population given the similar symptom burden and illness severity. For example, a study was conducted to monitor symptoms of patients who were undergoing chemotherapy due to advanced tumors [13]. The Symptom Tracking and Reporting (STAR), a web-based tool addressing 12 common symptoms monitored weekly, was implemented and its effect on HRQOL, survival, quality-adjusted survival, ER visits, and hospitalizations was assessed. If a symptom was reported to have worsened by 2 or more points, the nurses immediately received an email alert and would determine a course of action. This intervention was compared to usual care in which patients had conversations with their oncologists during clinical visits and received encouragement to reach out with symptom problems [13]. After a 6-month study period, patients who underwent the intervention had better HRQOL, fewer ER visits, few- 
er hospitalizations, a longer duration of undergoing chemotherapy treatment, and better quality-adjusted survival [13]. Similarly, other research has shown the benefit of calls administered by nurse practitioners to check in on patients during their first 2 rounds of chemotherapy, something that could be helpful at the initiation of dialysis [14]. The intervention included an initial call designed to address questions about postchemotherapy medication 1-3 days after treatment and a second call, 4-6 days after chemotherapy, designed to check on symptoms that were more likely to have begun [14]. Although Traeger et al. did not find that symptom burden was lowered because of these calls, participants did report that they were satisfied with the intervention. They found the calls reassuring and convenient, both factors that may be helpful in the treatment of any cancer or chronic disease. Such an intervention may be particularly relevant and impactful for patients with advanced CKD and ESRD since they share similar symptom burden as the cancer patients, and are often similarly dealing with multiple coexisting and fluctuating symptoms such as pain, depression, fatigue, and anxiety.

\section{Impact of PRO Measurement on HRQOL}

The treatment of symptoms such as depression, pain, lack of sleep, and erectile dysfunction are not symptoms immediately tended to by nephrologists. However, since they play a role in HRQOL, it is important to give them attention and study ways of improving those areas of health. One study focused on depression, pain, and erectile dysfunction - prominent symptoms but not often studied or treated [15]. The interventions compared were a feedback arm where renal providers were informed of their patients' symptoms monthly and decided on course of treatment versus a nurse management arm which consisted of the assessment of symptoms monthly and a trained nurse generating a treatment plan [15]. They found no improvement in the symptoms for either arm of the study; however, they discovered that both resulted in a small but significant reduction in symptoms when compared to usual care [15]. These findings indicate there is much research to be done exploring treatments for the underlying symptoms dialysis patients suffer from. Although there was not a huge change in symptoms in prior studies, there was some, and continued research could lead to larger reductions in symptoms for patients and improvement in HRQOL.

Symptom burden contributes significantly to HRQOL for CKD patients, and a better characterization of these symptoms can help providers approach treatment in an effective way that allows for improved HRQOL. The most prevalent symptoms reported among those with ESRD have been fatigue or lack of energy, feeling drowsy, pain, dry skin, and pruritus [16]. Also, little is known about the symptom burden experienced by stage 4 and 5 CKD patients and those who are undergoing peritoneal dialysis; more studies are needed to investigate these populations. Effect of a treatment on potential improvements in HRQOL may be one of the most important factors that influences a patient's decision about treatment options, particularly dialysis modalities [17]. Additionally, it is important that symptoms that affect HRQOL can be effectively assessed because that could improve patientprovider communication about possible treatment plans to target specific physical symptoms. While generic HRQOL PRO tools are helpful, they often fall short when it comes to assessing individual symptoms. Although there are PRO tools that address dialysis symptoms more specifically, these tools are in need of improvements so that they may be use for clinical symptom evaluation and responsiveness to intervention [17].

\section{Integration of the Patient Perspective with Technology}

EHRs are one form of technological advancements that have the potential to create efficiency when sharing medical records, which is a valuable ability because it could improve patient and clinician communication [4]. Due to the current COVID-19 pandemic, EHRs have even more use when it comes to minimizing patient and clinician physical contact. Another consideration is the communication between the various professionals that a patient with CKD may interact with, such as nephrologists, pharmacists, nurses, dieticians, and other healthcare workers [4].

Telehealth is an approach that has been used in other disciplines such as oncology and GI, and strategies used in these disciplines could be adapted and integrated into nephrology research. Telehealth can be used for symptom monitoring, patient check-ins, emergency assistance, and other interventions. Novel online resources used in cancer research, such as the STAR for patients undergoing chemotherapy, when combined with nurse review and timely symptom management intervention, have shown success in improving patient experience and lessening patient suffering. Although this tool targets symptoms common for chemotherapy patients, such as appetite loss, constipa- 
tion, cough, fatigue, and nausea, a similar tool could be developed and used to target symptoms common among dialysis patients. However, there is still research that needs to be done in these areas to develop interventions that specifically address the needs of patients with kidney disease and will be feasible and acceptable to the patients.

Now that there is a push for accelerating the use of telemedicine due to the COVID-19 pandemic, understanding which technological strategies benefit patients should be a top priority. One study looked at 3 models of care delivery for irritable bowel disease to assess remission status as a primary outcome and HRQOL as a secondary outcome [18]. The study found that both web-based care and nurse-assisted telephone calls were associated with a reduction in outpatient visits, and web-based care resulted in less calls when compared to the other 2 groups [18]. Therefore, it was concluded that web-based care is an effective intervention for improving health outcomes in patients suffering from irritable bowel disease. This could be because medication adherence was better in the webbased group due to higher levels of communication and relevant adaptations to care, and interference with daily activities was reduced because social impairment improved [18]. The improvements in remission rates and social functioning led to patients experiencing greater satisfaction and directly correlated to significant changes in patient HRQOL [18]. Among patients with kidney disease, a similar web-based intervention may provide an excellent platform to monitor and address a myriad of physical and psychosocial symptoms, treatment-related side effects, medication adherence, and fluid and dietary restrictions. Due to the potential of web-based medical intervention to improve HRQOL, it is an important area of study and should be further explored among patients with CKD/ESKD.

There has been limited studies of electronic PRO research in nephrology and standards for PROs in clinical trials. One study investigated the effectiveness of webbased eHealth portal for delivery of care to patients receiving dialysis from home [19]. Standard at-home dialysis care includes calls for urgent or nonurgent changes as well as nursing support over the phone. In this study, patients were enrolled in an online eHealth portal where they could interact with trained nurses [19]. However, there was no significant difference measured for HRQOL or improvement in home dialysis care [19]. Another study examining the use of electronic PROs in patients receiving home dialysis care found that electronic PROs were useful for obtaining information that allowed for better person-centered care and clinicians asked more

Patient-Reported Outcomes in Kidney Disease questions about symptoms and were more involved [9]. However, the study was unable to determine whether the intervention would be better than usual care, necessitating more research in this area. Since PROs have such potential to direct attention toward improving HRQOL, it is critical that they are used properly and effectively in clinical trials. There is evidence to show that adherence to high-quality standards for PRO measures as well as the management of data that come from PROs is lacking [20]. In clinical trials of PROs and ePROs among those with $\mathrm{CKD}$, trialists should report $\mathrm{PRO}$ outcome selection, data collection methods, PRO data management, analysis plan, and approaches to data missingness.

\section{Conclusion}

PROs allow the patient to share information efficiently and effectively about their experience with chronic illness, without potential bias from third parties. PROs delivered at the point of care can give the patient the sense of support and reassurance they need that they are on the path to improving their current condition. Dialysis is a significant part of treatment for ESKD patients and it influences HRQOL; therefore, further research should aim to improve HRQOL for dialysis patients with the use of PROs. Telehealth and the use of electronic PROs can be used for symptom monitoring, patient check-ins, emergency assistance, and other interventions, which could be extremely important due to COVID-19 and the effort to minimize close physical patient contact.

\section{Statement of Ethics}

The work is exempt from Ethics Committee approval.

\section{Conflict of Interest Statement}

The authors have no relevant conflicts of interest to declare.

\section{Funding Sources}

The authors did not receive any funding.

\section{Author Contributions}

S.M., M.U., and M.J. had roles in conception and design, writing, and editing of this narrative review. 


\section{References}

1 Zimbudzi E, Lo C, Ranasinha S, Gallagher M, Fulcher G, Kerr PG, et al. Predictors of healthrelated quality of life in patients with Co-Morbid diabetes and chronic kidney disease. PLoS One. 2016;11(12): 0168491.

2 Schick-Makaroff K, Thummapol O, Thompson S, Flynn R, Karimi-Dehkordi M, Klarenbach S, et al. Strategies for incorporating patient-reported outcomes in the care of people with chronic kidney disease (PRO kidney): a protocol for a realist synthesis. Syst Rev. 2019 12;8(1):20.

$3 \mathrm{Ju} \mathrm{A}$, Tong A. Considerations and challenges in selecting patient-reported outcome measures for clinical trials in nephrology. Clin J Am Soc Nephrol. 2017 7;12(11):1882-4.

4 Drawz PE, Archdeacon P, McDonald CJ, Powe NR, Smith KA, Norton J, et al. CKD as a model for improving chronic disease care through electronic health records. Clin J Am Soc Nephrol. 2015 Aug;10(8):1488-99.

5 Kefale B, Alebachew M, Tadesse Y, Engidawork E. Quality of life and its predictors among patients with chronic kidney disease: a hospital-based cross sectional study. PLoS One. 2019;14(2):e0212184.

6 Aiyegbusi OL, Kyte D, Cockwell P, Marshall T, Gheorghe A, Keeley T, et al. Measurement properties of patient-reported outcome measures (PROMs) used in adult patients with chronic kidney disease: a systematic review. PLoS One. 2017 Jun;12(6):e0179733.

7 Anderson NE, Calvert M, Cockwell P, Dutton M, Aiyegbusi OL, Kyte D. Using patient-reported outcome measures (PROMs) to promote quality of care in the management of patients with established kidney disease re- quiring treatment with haemodialysis in the UK (PROM-HD): a qualitative study protocol. BMJ Open. 2018 Oct;8(10):e021532.

8 Chong K, Unruh M. Why does quality of life remain an under-investigated issue in chronic kidney disease and why is it rarely set as an outcome measure in trials in this population? Nephrol Dial Transplant. 2017 Apr;32(Suppl 2):ii47-52.

9 Schick-Makaroff K, Tate K, Molzahn A. Use of electronic patient reported outcomes in clinical nephrology practice: a qualitative pilot study. Can J Kidney Health Dis. 2019;6: 2054358119879451.

10 Black N. Patient reported outcome measures could help transform healthcare. BMJ. 2013 Jan;346:f167.

11 Jardine MJ, Zuo L, Gray NA, de Zoysa JR, Chan CT, Gallagher MP, et al. A trial of extending hemodialysis hours and quality of life. J Am Soc Nephrol. 2017 Jun;28(6):1898911.

12 Mehrotra R, Cukor D, Unruh M, Rue T, Heagerty $\mathrm{P}$, Cohen SD, et al. Comparative efficacy of therapies for treatment of depression for patients undergoing maintenance hemodialysis: a randomized clinical trial. Ann Intern Med. 2019 Feb;170(6):369-79.

13 Basch E, Deal AM, Kris MG, Scher HI, Hudis CA, Sabbatini P, et al. Symptom monitoring with patient-reported outcomes during routine cancer treatment: a randomized controlled trial. J Clin Oncol. 2016 Feb;34(6):557-65.

14 Traeger L, McDonnell TM, McCarty CE, Greer JA, El-Jawahri A, Temel JS. Nursing intervention to enhance outpatient chemotherapy symptom management: patient-reported outcomes of a randomized controlled trial. Cancer. 2015 Nov;121(21):3905-13.

15 Weisbord SD, Mor MK, Green JA, Sevick MA, Shields AM, Zhao X, et al. Comparison of symptom management strategies for pain, erectile dysfunction, and depression in patients receiving chronic hemodialysis: a cluster randomized effectiveness trial. Clin J Am Soc Nephrol. 2013 Jan;8(1):90-9.

16 Almutary H, Bonner A, Douglas C. Symptom burden in chronic kidney disease: a review of recent literature. J Ren Care. 2013;39(3):14050.

17 Flythe JE, Powell JD, Poulton CJ, Westreich KD, Handler L, Reeve BB, et al. Patient-reported outcome instruments for physical symptoms among patients receiving maintenance dialysis: a systematic review. Am J Kidney Dis. 2015 Dec;66(6):1033-46.

18 Del Hoyo J, Nos P, Faubel R, Muñoz D, Domínguez D, Bastida G, et al. A web-based telemanagement system for improving disease activity and quality of life in patients with complex inflammatory bowel disease: pilot randomized controlled trial. J Med Internet Res. 2018 27;20(11):e11602.

19 Kiberd J, Khan U, Stockman C, Radhakrishnan A, Phillips M, Kiberd BA, et al. Effectiveness of a web-based eHealth portal for delivery of care to home dialysis patients: a singlearm pilot study. Can J Kidney Health Dis. 2018;5:2054358118794415.

20 Kyte DG, Draper H, Ives J, Liles C, Gheorghe A, Calvert M. Patient reported outcomes (PROs) in clinical trials: is 'In-Trial' guidance lacking? A systematic review. PLoS One. 2013 Apr;8(4):e60684. 